\title{
Should the management of high grade cervical squamous intraepithelial lesion (HSIL) be different in HIV-positive women?
}

\author{
Christine Gilles $^{1^{*}}$ (D), Maude Velghe-lenelle ${ }^{1}$, Yannick Manigart ${ }^{1}$, Déborah Konopnicki ${ }^{2}$ and Serge Rozenberg ${ }^{1}$
}

\begin{abstract}
Background: This study compares the management and outcome of high grade squamous intraepithelial lesions (HSIL) in HIV-positive and -negative women and identifies risk factors for treatment failure.

Methods: This retrospective, controlled study includes 146 HIV-positive women, matched for HSIL, age and year of diagnosis, with 146 HIV-negative women. Differences were analysed using parametric and non-parametric tests and Kaplan-Meier survival curves. A binary logistic regression was used to assess risk factors for treatment failure.

Results: Persistence of cervical disease was observed most frequently in HIV-positive women (42 versus 17\%) ( $p<$ 0.001 ) and the cone biopsy margins were more often invaded in HIV-positive-women than in HIV-negative ones. (37 versus $16 \% ; \mathrm{p}<0.05)$.

HIV-positive women, with successful cervical treatment had better HIV disease control: with significantly longer periods of undetectable HIV viral loads (VL) (19 versus 5 months; $p<0.001$ ) and higher CD4 counts (491 versus 320 cells/ $\left.\mathrm{mm}^{3} ; \mathrm{p}<0.001\right)$. HIV-positive women with detectable VL at the time of dysplasia had 3.5 times (95\% IC: 1.5-8.3) increased risk of treatment failure. Being treated through ablative therapy was associated with a 7.4 , four-fold (95\% IC: 3.2-17.3) increased risk of treatment failure compared to conization

Conclusion: HIV-positive women have a higher risk of treatment failure of HSIL than do HIV-negative women, especially when ablative therapy is used and in women with poor control of their HIV infection. The management and the follow- up of HSIL's guidelines in this high-risk population should be adapted consequently: for HIV-positive women with uncontrolled viral load, excisional treatment should be the preferred therapy, whereas for women with undetectable viral load, CD4 + lymphocytes higher than 500 cells $/ \mathrm{mm}^{3}$ and with a desire of pregnancy, ablative therapy may be considered.
\end{abstract}

Keywords: HIV, HSIL, Treatment failure, CIN

\section{Background}

It was estimated in 2018 , that 40 million people worldwide were living with HIV, of which about half were women [1]. The same year, it was estimated that there

\footnotetext{
*Correspondence: christine.gilles@stpierre-bru.be

${ }^{1}$ Department of Obstetrics and Gynecology, Saint Pierre University

Hospital Brussels, Université Libre de Bruxelles (ULB), Free University of Brussels (ULB-VUB), 322 Rue Haute, 1000 Brussels, Belgium

Full list of author information is available at the end of the article
}

were 570,000 new cases of cervical cancer, which represents $6.6 \%$ of all female cancers, making it the fourth most frequent cancer affecting women [2].

In 1993, the Centre for Disease Control and Prevention (CDC) concluded that HIV infected women, diagnosed with moderate or severe cervical intraepithelial neoplasia (CIN), should be viewed as suffering from an early stage, symptomatic HIV infection (category B), and those with invasive cervical cancer should be viewed as suffering 
from acquired immunodeficiency (AIDS; category C) [3]. Being infected with HIV is a risk factor for acquiring HPV infection, developing a persistence of HPV with subsequent CIN, or even for cervical cancer $[4,5]$. Consequently, HIV-positive women have on average a three times higher incidence of cervical lesions than HIV-negative women [6].

In 2005, we compared HIV-positive and negative women after treatment of CIN 2 and CIN 3 [CIN2 + or high grade intraepithelial squamous lesions (HSIL)] and reported that HIV positive women had higher rates of recurrence of CIN after a median follow-up of 22 months [7], which was confirmed in a systematic review [8].

At that time, many patients were not optimally treated as they were not using Combined Antiretroviral Therapy (cART), due to compliance issues (such as multiple pills and intakes or side effects). Moreover, many patients had been treated for only short periods of time [7]. Since then, compliance friendly cART (including one pill per day) has become widely available [9].

The primary aim of this study is to compare the management and the outcome of HSIL, for HIV-positive and HIV-negative women. The secondary aims were to address failure and success differences in relation to the chosen treatment (conization which refers to the excision of a cone-shaped portion of the cervix versus ablative therapy which refers to cryotherapy or electrocoagulation) and to determine risk factors for failures within the group of HIV positive women.

\section{Materials and methods Design}

We conducted a retrospective matched controlled study.

\section{Selection of the population}

Using the pathology data bank from our hospital, we identified all patients who had been diagnosed with CIN2 + (CIN 2 or CIN 3 or both, defined as HSIL) at biopsy or from conization specimens, between January 2003 and December 2017 ( $n=2669)$. We cross-matched this list with the data-bank of our HIV reference centre, identifying HIV positive women $(n=161)$. Fifteen patients were excluded ( 3 who had cervical cancer and 12 with no follow-up, resulting in the selection of $146 \mathrm{HIV}$ positive women who were included in this study (Fig. 1). We matched them with HIV negative women, found in the initial data-base, using the following matching criteria: CIN2 +, age, and year of diagnosis and without knowledge of any other item. At least one follow-up cervical smear after management was required for all the patients (Fig. 1).

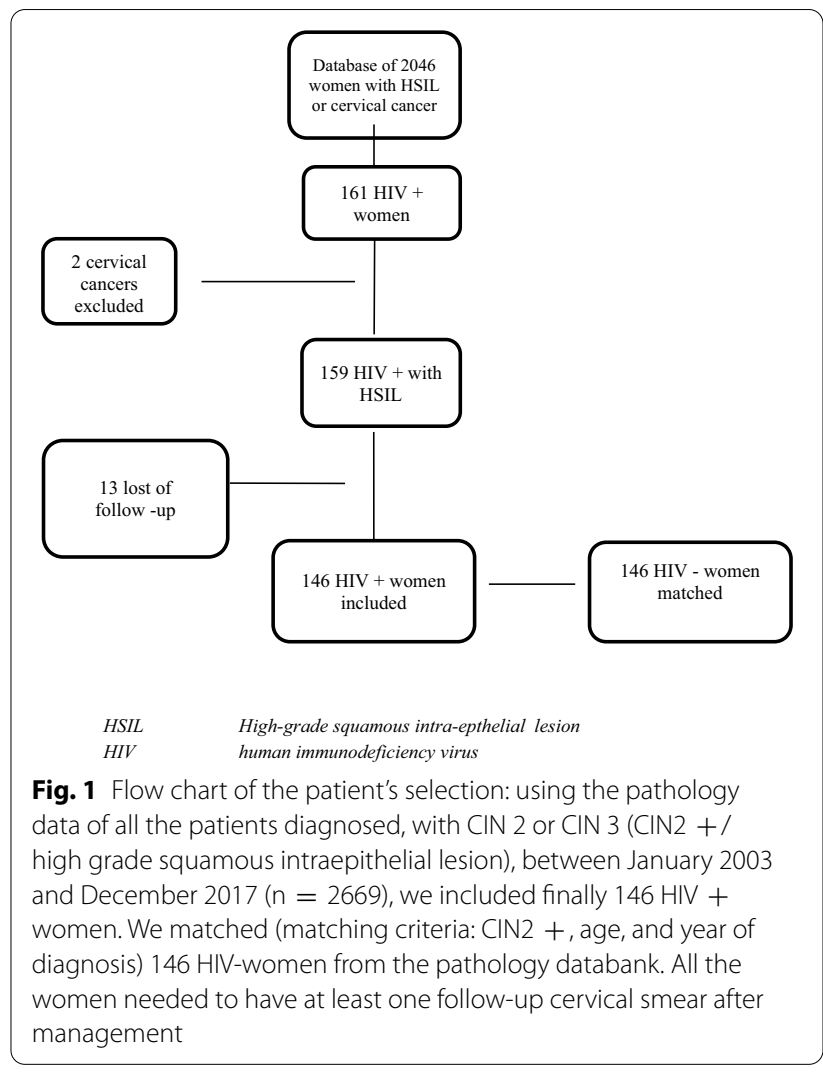

\section{Data collection}

We collected the following socio-demographical data from the computerised medical files: ethnic origin, access to health coverage, tobacco use, obstetrical history and parity, past and present contraceptive use and method "[IUD, combined hormonal contraception (pill, patch, ring), progestogen containing contraception (pill, injectable, implant), condom]".

STI history (syphilis, herpes, chlamydia trachomatis, neisseria gonorrhoea, hepatitis b), HPV vaccination history.

\section{Data pertaining to the cervical lesion}

Date of treatment, type of treatment (follow-up, cone biopsy or also known as conization (all excisional procedures were performed using loop electrosurgical excision procedure (LEEP), local treatment (application of trichloroacetic acid, imiquimod or podophylin), ablative therapy (cryotherapy, electrocoagulation) hysterectomy), the pathology results of the specimen (normal, low grade squamous intraepithelial lesion, LSIL, or HSIL for high grade squamous intraepithelial lesion), characteristics of conization specimen (whether the margins were free of disease or not (without distinction of whether it was endocervical or exocervical), its size, weight and volume), HPV genotyping results when available, it was expressed 
differently over time: either expressed as HRHPV + (if any of the following was positive $16 / 18 / 31 / 33 / 35 / 39 / 45 /$ $51 / 52 / 56 / 58 / 59 / 68)$ without precise identification of the genotype by Hybrid captur, (hc2 High-Risk HPV DNA Test, Digene ${ }^{\circledR}$, USA) or either expressed as HPV 16 or $18+$ or other than 16 and $18(31 / 33 / 35 / 39 / 45 / 51 / 52 / 56 /$ 58/59/68) performed by PCR (Abbott HRHPV ${ }^{\circledR}$ ).

\section{Follow-up data}

Date and result of every follow-up cervical smear, HPV testing and/or cervical biopsy results, duration of the follow-up (in weeks). We defined persistence as the presence of an abnormal smear (ASCUS HPV HR +, LSIL, HSIL) at the first follow- up visit. We defined a recurrence as being an HSIL lesion diagnosed by biopsy, during follow-up after having had a previous, normal cervical result. We defined as treatment failure when a persistence or a recurrence was observed.

\section{HIV infection data}

Age at time of HIV diagnosis, CD4 count rate (cells $/ \mathrm{mm}^{3}$ ) and viral load (copies/ml) at time of HIV diagnosis and at time of cervical lesion diagnosis, risk factors for HIV transmission (heterosexual, intravenous drug use, transfusion, mother-to-child), cART type (Nucleoside analog reverse-transcriptase inhibitors, Protease Inhibitors, Non-nucleoside reverse-transcriptase inhibitors, Integrase Inhibitors)and intake., duration of cART until the dysplasia was discovered (length of time during which cART was used in months) and effective duration of cART taking into account treatment compliance (cumulative length of time during which cART was used in months), AIDS stage according to the CDC classification $(\mathrm{A}, \mathrm{B}, \mathrm{C}), \mathrm{CD} 4 / \mathrm{CD} 8$ at the time of cervical lesion diagnosis; maximum viral load (copies/ml) and CD4 nadir $\left(\right.$ cells $/ \mathrm{mm}^{3}$ ) between HIV diagnosis and cervical lesion diagnosis; cumulative time of viral load $<50$ copies $/ \mathrm{ml}$.

\section{Statistical analyses}

Descriptive statistics were analysed. Normal distribution was checked by visual histograms and using the Shapiro-Wilk test $(\mathrm{p}<0.05)$. Differences in the distribution of variables were tested for independent groups using the Student $\mathrm{T}$ test that were normally distributed or using Wilcoxon-Mann-Whitney test for variables that were not normally distributed, since we were unable to match the patients for ethnicity. Differences in proportions were tested using Khi Square teste and exact de Fisher correction for small numbers; Kaplan-Meier analysis was used to assess persistence and recurrence in relation to time (days without recurrence or persistence). Differences between groups were tested using the Log-rank test.

A binary logistic regression was used to assess risk factors of persistence, expressed as Odd Ratios (OR) with a 95\% confidence interval. A generalized linear regression model was used to assess the analysis of variance for multiple dependent variables. Cox-regression was used to estimate Hazard ratios.

All analyses were performed using SPSS (IBM statistics 25) or SAS.

The study was approved on March 20th, 2018 by the Saint-Pierre University Hospital Ethical Review Board (nr CE/18-03-08).

\section{Results}

\section{Population characteristics (Table 1)}

The HIV infected women who came mostly from subSaharan Africa, were more frequently unemployed, had less health-insurance coverage, were less educated and smoked less than HIV-negative women (Table 1). Twenty-six percent of HIV-positive women used condoms compared to $3 \%$ of the negative ones. There was a higher prevalence of sexually transmitted infections in the group of HIV positive women (27\%) than in HIV negative ones (4\%).

\section{Cervical lesion data (Table 2)}

There were no differences between the two groups in terms of HSIL management: respectively $53 \%$ of HIVpositive and $64 \%$ of HIV-negative women underwent a cone biopsy and $33 \%$ of HIV positive and $25 \%$ of HIV negative women were treated by topical therapy (which includes ablative therapy or local application of trichloroacetic acid, podophylin or imiquimod). Persistence of cervical disease was more frequently observed in HIV-positive women (41.8\%, of which $47.5 \%$ had a HSIL lesion) than in HIV negative ones (16.5\%, of which $41.6 \%$ had a HSIL; p < 0.001). Median time to persistence was 63 days (IQR: 6-172). There was no difference between the two groups in terms of recurrence.

The duration of follow- up was comparable in the two groups (165 weeks in HIV-positive versus 142 weeks in HIV-negative). The number of follow-up visits were the same between the two groups with a median of 7 (IQR: 1-17). Seventeen women (9 HIV-negative, 8 HIV-positive) were vaccinated against HPV (details were missing about the vaccine type); this data was missing for 183 patients. 
Table 1 Demographic and clinical data of HIV negative and positive women with HSIL

\begin{tabular}{|c|c|c|c|c|}
\hline & $\begin{array}{l}\text { Evaluated population (total } \\
N=292)^{c}\end{array}$ & $H I V-(N=146)$ & $\mathrm{HIV}+(\mathrm{N}=146)$ & $P$ value \\
\hline \multicolumn{5}{|l|}{ Age } \\
\hline Median (IQR) & 292 & $35(29-41)$ & $35(29-41)$ & $0.944^{\mathrm{a}}$ \\
\hline Origin & 269 & $N=123$ & $N=146$ & $<0.001^{b}$ \\
\hline Europe & 92 & 76 (61.8\%) & $16(10.9 \%)$ & \\
\hline North Africa & 30 & $29(23.6 \%)$ & $1(0.7 \%)$ & \\
\hline Sub-Sahara Africa & 140 & $12(9.8 \%)$ & $128(87.7 \%)$ & \\
\hline Asia & 1 & $1(0.8 \%)$ & $0(0 \%)$ & \\
\hline South America & 6 & $5(4.1 \%)$ & $1(0.7 \%)$ & \\
\hline Level of education & 76 & $N=18$ & $N=58$ & $0.058^{b}$ \\
\hline Primary school & 15 & $3(16.7 \%)$ & $12(20.7 \%)$ & \\
\hline Secondary school & 36 & $5(27.8 \%)$ & $32(53.4 \%)$ & \\
\hline Higher education & 25 & $10(55.6 \%)$ & $15(25.7 \%)$ & \\
\hline Employment & 141 & $N=42$ & $N=99$ & $<0.001^{b}$ \\
\hline Unemployed & 85 & $14(33.3 \%)$ & $71(71.7 \%)$ & \\
\hline Employed & 49 & $25(59.5 \%)$ & $24(24.2 \%)$ & \\
\hline Student & 4 & $2(4.8 \%)$ & $2(2 \%)$ & \\
\hline Retired & 3 & $1(2.4 \%)$ & $2(2 \%)$ & \\
\hline Legal status & 227 & $N=83$ & $N=144$ & $0.011^{b}$ \\
\hline Legally in Belgium & 196 & $78(94 \%)$ & $118(81.9 \%)$ & \\
\hline No residence permit & 31 & $5(6 \%)$ & $26(18.1 \%)$ & \\
\hline Access to health care & 288 & $N=142$ & $N=146$ & $<0.001^{b}$ \\
\hline None & 11 & $1(0.7 \%)$ & $10(6.8 \%)$ & \\
\hline Medical regular insurance & 237 & $130(91.5 \%)$ & $107(73.3 \%)$ & \\
\hline Social assistance & 40 & $11(7.7 \%)$ & $29(19.9 \%)$ & \\
\hline Smoking & 258 & $N=121$ & $N=137$ & $<0.001^{b}$ \\
\hline Non-smoker & 176 & $65(53.7 \%)$ & $111(81 \%)$ & \\
\hline Smoker & 82 & $56(46.3 \%)$ & $26(19 \%)$ & \\
\hline Contraception & 270 & $N=138$ & $N=132$ & $<0.001^{b}$ \\
\hline None & 122 & $62(44.9 \%)$ & $60(45.5 \%)$ & \\
\hline Hormonal contraception & 80 & $51(36.9 \%)$ & $29(21.9 \%)$ & \\
\hline Non hormonal contraception & 68 & $25(18.2)$ & $43(32.6)$ & \\
\hline Past history of STI & 290 & $N=144$ & $N=146$ & $<0.001^{b}$ \\
\hline Not reported & 244 & $138(95.8 \%)$ & $106(72.6 \%)$ & \\
\hline At least one episode of STI & 46 & $6(4.2 \%)$ & $40(27.4 \%)$ & \\
\hline Parity & 289 & 143 & 146 & $0.024^{\mathrm{a}}$ \\
\hline Median (IQR) & & $1(0-2)$ & $1(1-3)$ & \\
\hline
\end{tabular}

Hormonal contraception includes estro-progestins (pill, ring, patch), progestin methods include (progestin only pill, implant, injectable) and the use of concomitant hormonal method and condoms

Non hormonal contraception includes IUD, condoms and sterilization

$H I V$ human immunodeficiency virus; STI sexually transmitted infection; HSIL high grade squamous intraepithelial lesion; IQR interquartile range

a Mann-Whitney test

${ }^{\mathrm{b}}$ khi-square test

' Some analyses were realised on a restricted number of patients. These numbers are provided in the column evaluated population

\section{Cone biopsy data (Table 3)}

High grade lesions were confirmed in cones, respectively, in 90 and $92 \%$ of the specimens from HIV + and HIV - women. The margins of the specimens were more frequently positive in HIV-positive women than in HIV negative ones (37 versus $16 \%$; $<$ 0.05). There were no differences in volume, depth nor weight of the biopsy specimens between the two groups. 
Table 2 Cervical lesion, management and follow up of HIV negative and positive women

\begin{tabular}{|c|c|c|c|c|}
\hline & $\begin{array}{l}\text { Evaluated population } \mathrm{N}= \\
292^{\mathrm{d}}\end{array}$ & $H I V-(N=146)$ & $\mathrm{HIV}+(\mathrm{N}=146)$ & P value \\
\hline Baseline pap-test & 286 & $N=140(100 \%)$ & $N=146(100 \%)$ & $0.010^{b}$ \\
\hline Normal & 1 & $0(0 \%)$ & $1(0.7 \%)$ & \\
\hline LSIL & 90 & $38(27.1 \%)$ & $52(35.6 \%)$ & \\
\hline HSIL & 152 & $73(52.1 \%)$ & $79(54.1 \%)$ & \\
\hline ASCUS & 35 & $22(15 \%)$ & $14(9.6 \%)$ & \\
\hline $\mathrm{ASCH}$ & 7 & $7(5 \%)$ & $0(0 \%)$ & \\
\hline Cancer & 1 & $1(0.7 \%)$ & $0(0 \%)$ & \\
\hline HPV & 141 & $N=42$ & $N=99$ & $0.325^{b}$ \\
\hline Negative & 9 & $2(4.8 \%)$ & $7(7.1 \%)$ & \\
\hline HR HPV & 108 & $31(73.8 \%)$ & $77(77.8 \%)$ & \\
\hline HPV 16/18 & 8 & $5(11.9 \%)$ & $3(3 \%)$ & \\
\hline HPV other than $16 / 18$ & 13 & $3(7.1 \%)$ & $10(10.1 \%)$ & \\
\hline HPV 16 and other & 3 & $1(2.4 \%)$ & $2(2 \%)$ & \\
\hline Baseline biopsy & 284 & $N=140(100 \%)$ & $N=144(100 \%)$ & $0.856^{b}$ \\
\hline Normal $^{c}$ & 4 & $2(1.4 \%)$ & $2(1.4 \%)$ & \\
\hline$L S I L^{c}$ & 3 & $2(1.4 \%)$ & $1(0.7 \%)$ & \\
\hline HSIL & 277 & $136(97.1 \%)$ & $141(97.9 \%)$ & \\
\hline Vaccination & 280 & $N=134$ & $N=146$ & $0.791^{b}$ \\
\hline Unvaccinated & 80 & $40(29.8 \%)$ & $40(27.4 \%)$ & \\
\hline Vaccinated & 17 & $9(6.7 \%)$ & $8(5.5 \%)$ & \\
\hline Not reported & 183 & $85(63.4 \%)$ & $98(67.1 \%)$ & \\
\hline Treatment & 292 & $N=146(100 \%)$ & $N=146(100 \%)$ & $0.290^{b}$ \\
\hline Follow-up & 27 & $12(8.2 \%)$ & $15(10.3 \%)$ & \\
\hline Conisation & 172 & $94(64.4 \%)$ & $78(53.4 \%)$ & \\
\hline Ablative treatment or local therapy & 85 & $37(25.3 \%)$ & $48(32.9 \%)$ & \\
\hline Hysterectomy & 8 & $3(2 \%)$ & $5(3.5 \%)$ & \\
\hline Persistence & 292 & $N=146(100 \%)$ & $N=146(100 \%)$ & $<0.001^{b}$ \\
\hline No & 207 & $122(83.5 \%)$ & $85(58.2 \%)$ & \\
\hline Yes & 85 & $24(16.5 \%)$ & $61(41.8 \%)$ & \\
\hline Type of persistence & 85 & $N=24(100 \%)$ & $N=61(100 \%)$ & $0.072^{b}$ \\
\hline CIN1 & 32 & $9(37.5 \%)$ & $28(45.9 \%)$ & \\
\hline $\mathrm{CIN} 2 / \mathrm{CIN} 3$ & 35 & $10(41.6 \%)$ & $29(47.5 \%)$ & \\
\hline ASCUS HPV + & 5 & $4(16.6 \%)$ & $1(1.6 \%)$ & \\
\hline $\mathrm{ASCH}$ & 4 & $1(4.3 \%)$ & $3(5 \%)$ & \\
\hline Recurrence & 292 & $N=146(100 \%)$ & $N=146(100 \%)$ & $0.18^{b}$ \\
\hline No & 277 & $136(93.1 \%)$ & $141(96.5 \%)$ & \\
\hline Yes & 15 & $10(6.9 \%)$ & $5(3.5 \%)$ & \\
\hline \multicolumn{5}{|l|}{ Time of follow-up (in weeks) } \\
\hline Median (IQR) & 292 & $142(62-304)$ & $165(48-444)$ & $0.112^{\mathrm{a}}$ \\
\hline
\end{tabular}

HIV human immunodeficiency virus; HPV human papillomavirus; LSIL low grade squamous intraepithelial lesion; HSIL high grade squamous intraepithelial lesion; ASCUS atypical squamous cell of undetermined significance; $A S C H$ atypical squamous cells that cannot exclude HSIL; HRHPV high risk human papillomavirus; IQR interquartile range; $C I N$ cervical intraepithelial neoplasia

a Mann-Whitney test

${ }^{b}$ khi-carré test

'These patients had HSIL diagnosis at time of conization

${ }^{d}$ Some analyses were realised on a restricted number of patients. These numbers are provided in the column evaluated population 
Table 3 Data concerning the conisation specimen in HIV + and - women

\begin{tabular}{llll}
\hline & $\mathbf{N}=\mathbf{1 7 2}$ & HIV $-(\mathbf{N}=\mathbf{9 4})$ & HIV + (N= 78) \\
\hline Pathology report & 170 & $\mathrm{~N}=92(100 \%)$ & $\mathrm{N}=78(100 \%)$ \\
Free of disease & 7 & $2(2.2 \%)$ & $5(6.4 \%)$ \\
LSIL & 8 & $5(5.4 \%)$ & $3(3.8 \%)$ \\
HSIL & 155 & $85(92.4 \%)$ & $70(89.7 \%)$ \\
Excision margins & 171 & $\mathrm{~N}=93(100 \%)$ & $\mathrm{N}=78(100 \%)$ \\
Free of disease & 127 & $78(83.9 \%)$ & $49(62.8 \%)$ \\
Involved & 44 & $15(16.1 \%)$ & $29(37.2 \%)$ \\
Weight of the specimen (gr) & 56 & $\mathrm{~N}=35$ & $\mathrm{~N}=21$ \\
Median (IQR) & $2.48(1.8-3.9)$ & $2.4(1.77-4.5)$ & $2.55(1.91-3.36)$ \\
Depth of the specimen (cm) & 166 & $\mathrm{~N}=92$ & $\mathrm{~N}=74$ \\
Median (IQR) & $1.2(1-1.6)$ & $1.25(1-1.6)$ & $1.15(1-1.52)$ \\
Volume the specimen $\left(\mathrm{cm}^{3}\right)$ & 166 & $\mathrm{~N}=92$ & $\mathrm{~N}=74$ \\
Median (IQR) & $4.2(2.45-6.81)$ & $4.5(2.47-7.34)$ & $4(2.4-5.8)$ \\
\hline
\end{tabular}

LSIL low grade squamous intraepithelial lesion; HSIL high grade squamous intraepithelial lesion

${ }^{a}$ Mann-Whitney test

${ }^{b}$ khi-Square test

\section{Characteristics of the HIV-positive women (Table 4)}

Most HIV positive women had been infected through heterosexual contact and had been diagnosed with HIV at a median age of 28 years. Eighty-two percent had used cART, on average, for 34 months and $15 \%$ had already reached AIDS stage $C$ (Table 4). Among women under cART, the most used cARTs were 2 NRTI (Nucleoside analog reverse-transcriptase inhibitors +1 PI (Protease Inhibitors; 54.8\%), 2 NRTI + 1 NNRTI (Non-nucleoside reverse-transcriptase inhibitors (23.3\%) and 2 NRTI +1 INTI (Integrase Inhibitos; 6.4\%).

HIV-positive women, with successful cervical treatment had better HIV disease control: they had longer period of undetectable viral load (VL) (19 versus 5 months; $\mathrm{p}<0.001)$ than those with treatment failure. They also more frequently had VL $<50$ copies/ml at the time of the cervical treatment (69 versus $47 \%$; $\mathrm{p}=$ 0.008 ), lower peak VL between time of HIV diagnosis and cervical treatment (28,000 versus $1,00,000$ copies/ $\mathrm{ml} ; \mathrm{p}=0.002)$, higher nadir CD4 counts (230 versus 168 cells $/ \mathrm{mm}^{3} ; \mathrm{p}=0.01$ ) and a CD4/CD8 ratio that was significantly higher $(0.6$ versus $0.32 ; \mathrm{p}<0.001)$.

Median CD4 count at time of dysplasia was 491 CD4 + -lymphocytes cells $/ \mathrm{mm}^{3}$ in women with no treatment failure versus 320 in women with failure ( $\mathrm{p}<$ 0.01). HIV-positive women who developed recurrence or persistence had been more often managed with local or ablative therapy $(\mathrm{p}<0.05)$. We analysed the odds ratio of having treatment failure among HIV patients. A multivariate analysis showed that HIV-positive women with a VL $>50$ copies at the time of dysplasia had 3, 5-fold (95\% IC: 1.5-8.3) increased risk of treatment failure $(\mathrm{p}=0.004)$. Independently, being treated with local or ablative therapy rather than having had a conization was associated with a 7.4, four-fold (95\% IC: $3.2-17.3$ ) raised risk of having a treatment failure ( $\mathrm{p}<$ $0.001)$.

We analysed treatment failure among HIV-positive women versus HIV-negative ones using the KaplanMeier survival curve and observed that HIV-positive women had more recurrence or persistence than HIVnegative ones. (Log-rank test $=0.008$ ). Mean duration of time to treatment failure was $461 \pm 31$ days in $\mathrm{HIV}+$ women versus $620 \pm 39$ days in HIV negative ones (Fig. 2).

We also analyzed treatment failure in relation to the type of treatment (ablative, local or excisional) and observed that the risk of treatment failure is higher when ablative or local therapy is used compared to excisional procedures (log-rank test $<0.001)$. Mean time to recurrence or persistence was $208 \pm 22$ days for ablative therapy, $211 \pm 42$ days for local therapy versus $695 \pm 29$ days for cone biopsy. (Fig. 3).

Being HIV positive was associated in the Cox regression model, with a significant increased risk of treatment failure (Hazard ratio 1.7; 95\% confidence interval $1.1-2.6 ; \mathrm{p}=0.012$ ). Similarly, ablative therapy was associated with an increased risk of treatment failure as compared to conization (Hazard ratio 1.2; 95\% confidence interval 1.0-1.4; $\mathrm{p}=0.012$ ). 
Table 4 Characteristics of the HIV infection at time of cervical dysplasia diagnosis in relationship with cervical treatment failure

\begin{tabular}{|c|c|c|c|c|}
\hline & HIV (all women) $\mathrm{N}=146$ & $\begin{array}{l}\text { HIV women with successful SIL } \\
\text { treatment } \mathrm{N}=80\end{array}$ & $\begin{array}{l}\text { HIV women with SIL } \\
\text { treatment failure } \mathrm{N}=66\end{array}$ & $P$ value \\
\hline \multicolumn{5}{|l|}{ Age at HIV diagnosis } \\
\hline $\mathrm{N}$ & 146 & 80 & 66 & \\
\hline Mean (SD) & 28 & $29.5(7.7)$ & $27.8(8.1)$ & $0.47^{\mathrm{a}}$ \\
\hline \multicolumn{5}{|l|}{ Risk factor for HIV acquisition } \\
\hline $\mathrm{N}$ & 137 & 75 (100\%) & $62(100 \%)$ & $0.09^{b}$ \\
\hline Blood transfusion & 2 & 0 & $2(3.2 \%)$ & \\
\hline Heterosexual & 130 & 74 (98.7) & $56(90.3)$ & \\
\hline IVD & 2 & $1(1.3 \%)$ & $1(1.6 \%)$ & \\
\hline Mother to child & 3 & 0 & $3(4.8 \%)$ & \\
\hline cART use & 146 & $80(100 \%)$ & $66(100 \%)$ & \\
\hline Yes & $124(84.9 \%)$ & $69(86.3 \%)$ & $55(83.3 \%)$ & $0.62^{b}$ \\
\hline No & $22(15.1 \%)$ & $11(13.7 \%)$ & $11(16.7 \%)$ & \\
\hline \multicolumn{5}{|c|}{ Duration of time using CART at time of dysplasia (month) } \\
\hline $\mathrm{N}$ & 124 & 69 & 55 & \\
\hline Median (IQR) & $34(10-85)$ & $35(11-82)$ & $27(8-104)$ & $0.95^{\mathrm{a}}$ \\
\hline \multicolumn{5}{|c|}{ Cumulative duration of time treated with CART (month) } \\
\hline $\mathrm{N}$ & 124 & 69 & 55 & \\
\hline Median(IQR) & $23(7-60)$ & $29(9-62)$ & $22(6-61)$ & $0.53^{\mathrm{a}}$ \\
\hline HIV classification (CDC) & 146 & $80(100 \%)$ & $66(100 \%)$ & \\
\hline$A$ and $B$ & $124(84.9 \%)$ & $70(87.5 \%)$ & $54(81.8 \%)$ & $0.34^{\mathrm{b}}$ \\
\hline C & $22(15.1 \%)$ & $10(12.5 \%)$ & $12(18.2 \%)$ & \\
\hline \multicolumn{5}{|c|}{ VL at time of dysplasia diagnosis (copies/ml) } \\
\hline $\mathrm{N}$ & 142 & 79 & 63 & \\
\hline Median(IQR) & $50(20-646)$ & $50(20-131)$ & $88(20-5990)$ & $0.02^{\mathrm{a}}$ \\
\hline$\leq 50$ & $85(59.9 \%)$ & $55(69.6 \%)$ & $30(47.6 \%)$ & $0.008^{b}$ \\
\hline$>50$ & $57(40.1 \%)$ & $24(30.4 \%)$ & $33(52.4 \%)$ & \\
\hline$\geq 10 \times 5$ & $31(37.8 \%)$ & $14(35 \%)$ & $17(40.5 \%)$ & \\
\hline \multicolumn{5}{|c|}{ Cumulative length of undetectable VL (month) } \\
\hline N & 125 & 68 & 57 & \\
\hline Median (IQR) & $12(3-26)$ & $19(7-42)$ & $5(0.5-17)$ & $<0.001^{\mathrm{a}}$ \\
\hline $\mathrm{N}$ & 107 & $63(100 \%)$ & $44(100 \%)$ & $0.04^{b}$ \\
\hline $\begin{array}{l}\text { Number of patient having unde- } \\
\text { tectable VL less than } 1 \text { year }\end{array}$ & 46 & $22(34.9 \%)$ & $24(54.9 \%)$ & \\
\hline $\begin{array}{l}\text { Number of patient having unde- } \\
\text { tectable VL more than } 1 \text { year }\end{array}$ & 61 & $41(65.1 \%)$ & $20(45.5 \%)$ & \\
\hline \multicolumn{5}{|l|}{ Maximal VL (copies/ml) } \\
\hline $\mathrm{N}$ & 145 & $79(100 \%)$ & $66(100 \%)$ & \\
\hline Median (IQR) & $64,600(6170-1,87,000)$ & $28,000(2550-1,20,000)$ & $1,00,000(1675-2,71,250)$ & $0.002^{\mathrm{a}}$ \\
\hline$<10 \times 5$ & $81(55.9 \%)$ & $52(65.8 \%)$ & $29(43.9 \%)$ & $0.08^{b}$ \\
\hline$\geq 10 \times 5$ & $64(44.1 \%)$ & $27(34.2 \%)$ & $37(56.1 \%)$ & \\
\hline \multicolumn{5}{|c|}{ Nadir CD4 at time of dysplasia (cells/mm³) } \\
\hline $\mathrm{N}$ & 145 & $79(100 \%)$ & $66(100 \%)$ & \\
\hline Median(IQR) & $207(102-317)$ & $230(171-345)$ & $168(78-290)$ & $0.01^{\mathrm{a}}$ \\
\hline$\geq 200$ & $75(51.7 \%)$ & $57(62.7 \%)$ & $28(37.3 \%)$ & $0.04^{b}$ \\
\hline$<200$ & $70(48.3 \%)$ & $33(40.5 \%)$ & $38(57.6 \%)$ & \\
\hline$\geq 100$ & $110(75.9 \%)$ & $66(83.5 \%)$ & $44(66.7 \%)$ & $0.01^{b}$ \\
\hline$<100$ & $35(24.1 \%)$ & $13(16.5 \%)$ & $22(33.3 \%)$ & \\
\hline$\geq 50$ & $124(85.5 \%)$ & $69(87.3 \%)$ & $55(83.3 \%)$ & $0.49^{b}$ \\
\hline$<50$ & $21(14.5 \%)$ & $10(12.7 \%)$ & $11(16.7 \%)$ & \\
\hline
\end{tabular}


Table 4 (continued)

\begin{tabular}{|c|c|c|c|c|}
\hline & HIV (all women) $\mathrm{N}=146$ & $\begin{array}{l}\text { HIV women with successful SIL } \\
\text { treatment } \mathrm{N}=80\end{array}$ & $\begin{array}{l}\text { HIV women with SIL } \\
\text { treatment failure } \mathrm{N}=66\end{array}$ & $P$ value \\
\hline \multicolumn{5}{|l|}{$\mathrm{CD} 4 / \mathrm{CD} 8$} \\
\hline N & 140 & 76 & 64 & \\
\hline Median (IQR) & $0.5(0.25-0.8)$ & $0.6(0.3-0.85)$ & $0.32(0.2-0.55)$ & $<0.001^{\mathrm{a}}$ \\
\hline \multicolumn{5}{|c|}{ CD4 at the time of the dysplasia (cells $/ \mathrm{mm}^{3}$ ) } \\
\hline $\mathrm{N}$ & 142 & $78(100 \%)$ & $64(100 \%)$ & \\
\hline Median (IQR) & $430(237-556)$ & $491(380-636)$ & $320(182-465)$ & $<0.001^{\mathrm{a}}$ \\
\hline $0-200$ & $28(19.7 \%)$ & $9(11 \%)$ & $19(29.7 \%)$ & $<0.001^{b}$ \\
\hline $200-349$ & $27(19 \%)$ & $9(11.5 \%)$ & $18(28.1 \%)$ & \\
\hline $350-499$ & $37(26.1 \%)$ & $23(29.5 \%)$ & $14(21.9 \%)$ & \\
\hline$>500$ & $50(35.2 \%)$ & $37(47.4 \%)$ & $13(20.3 \%)$ & \\
\hline \multicolumn{5}{|c|}{ Management of the cervical dysplasia } \\
\hline $\mathrm{N}$ & 146 & $80(100 \%)$ & $66(100 \%)$ & $<0.001^{b}$ \\
\hline Follow-up & $15(10.3 \%)$ & $3(3.8 \%)$ & $12(18.2 \%)$ & \\
\hline Conisation & 78 (53.4\%) & $59(73.8 \%)$ & $19(28.8 \%)$ & \\
\hline Ablative or local treatment & 48 32.9\%) & $14(17.5 \%)$ & $34(51.5 \%)$ & \\
\hline Hysterectomy & $5(6.5 \%)$ & $4(5 \%)$ & $1(1.5 \%)$ & \\
\hline
\end{tabular}

$V L$ viral load; IVD intravenous drug use; CART combination antiretroviral therapy; HIV human immunodeficiency virus

${ }^{a}$ Mann-Whitney test

${ }^{\mathrm{b}}$ khi-carré test

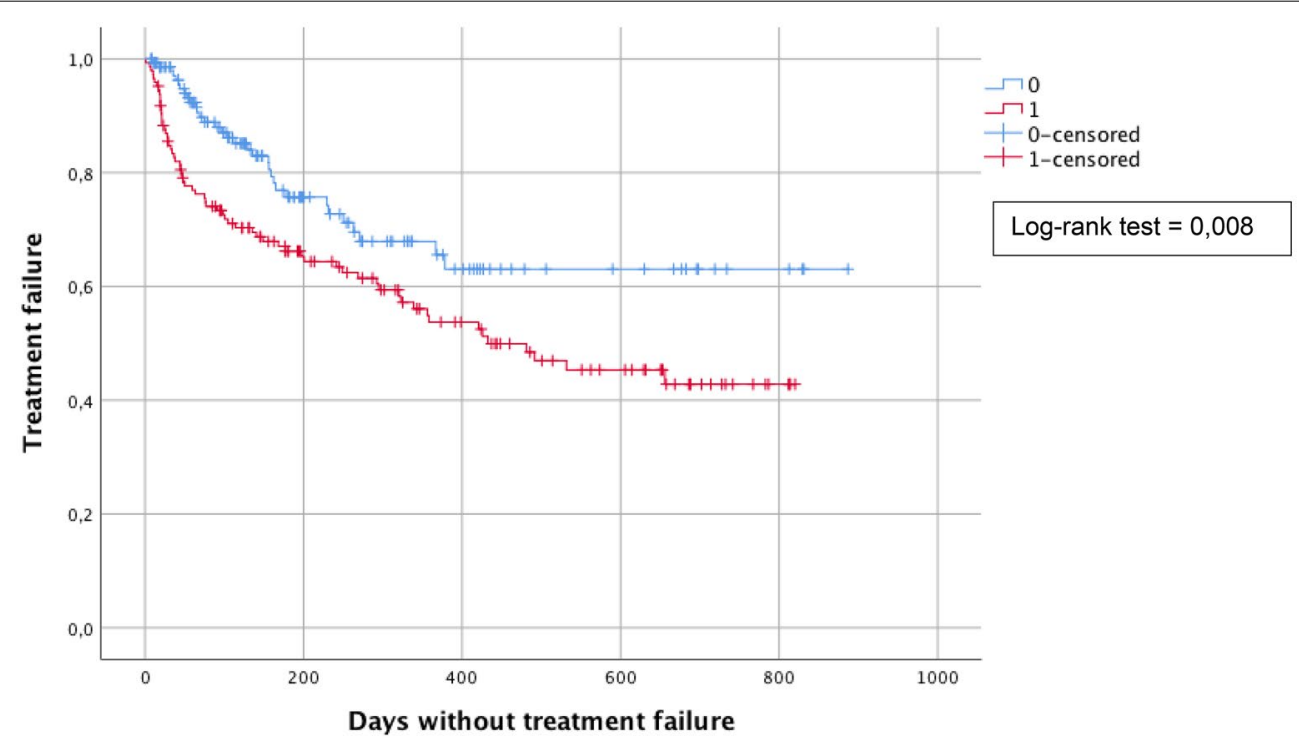

Fig. 2 Kaplan-Meier survival curves representing treatment failure in HIV-positive (blue line) and negative (red line) women. Crosses represent normal follow-up smears (censored $=$ no treatment failure) while decreases of the curve represent a treatment failure. HIV-positive women had more often treatment failure than HIV-negative ones. (log-rank test $=0.008$ )

\section{Discussion}

The aim of this study was to compare the management and outcome of high grade SIL in HIV-positive versus HIV-negative women.

Whereas the management of HSIL in the two groups was comparable, we observed that, after treatment of the cervical lesion, HIV-positive women had a 2.5 times higher risk of experiencing treatment failure. Our data are in concordance with a recent meta-analysis reporting that HIV-positive women had a two-fold higher risk of treatment failure compared to HIV negative women [8]. We also studied whether this treatment failure was due 


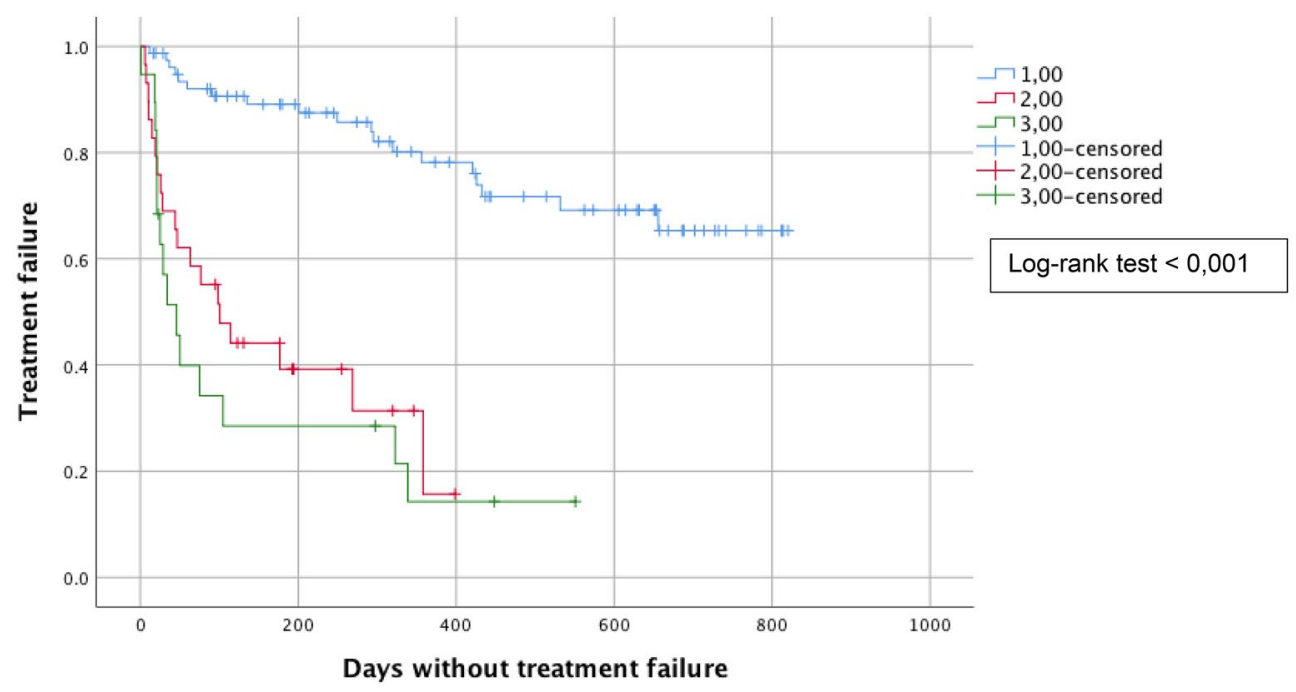

Fig. 3 Kaplan Meyer survival curves representing treatment failure in relation to the type of treatment (ablative in red/local in green or excisional treatment in blue) in the total combined population of HIV-positive and negative women. Crosses represent normal follow-up smears (censored $=$ no treatment failure) while decreases of the curve represent a treatment failure. The risk of treatment failure is higher when an ablative or local therapy is used compared to an excisional procedure (log-rank test $<0.001$ )

to either an increased persistence or an increased recurrence, rate. No differences were observed in terms of recurrence but 2.5 times increase in persistence risk was observed in HIV-positive women versus HIV-negative ones.

We observed, in both groups, a higher rate of treatment failure following local or ablative therapy than after LEEP. The data found in the literature are conflicting. In HIVnegative women, a meta-analysis comparing LEEP, cryotherapy and cold knife cone biopsy for HSIL, reported similar recurrence rates after 12 months, for LEEP and cryotherapy (5.3\%) and a recurrence rate of $1.4 \%$ using cold knife cone biopsy [10]. The authors of this metaanalysis also included observational studies because of the paucity of randomized controlled trials (RCT) [10]. Another meta-analysis, including four RCTs in HIVnegative and -positive women, comparing cryotherapy and LEEP for the treatment of CIN 1, 2 or 3, found that treatment with LEEP was associated with a lower risk of persistence at 6 months (RR 0.87, 95\% CI 0.76-0.99) and recurrence at 12 months (RR: $0.91,95 \%$ CI 0.84-0.99) compared to cryotherapy treatment [11].

In our study, HIV-positive women, who had been treated by local or ablative therapy, had an increased risk of treatment failure. Similarly, two RCTs in HIV positive women, reported that cryotherapy was associated with a higher rate of treatment failure than LEEP. Chirenje et al. [12] observed a treatment failure after cone biopsy of $4 \%(n=50)$ versus $14 \%$ after cryotherapy $(n=42)$ at the12 month follow-up. Green et al. [13] compared 200
HIV-positive women who had HSIL and were treated with cryotherapy, to 200 treated using cone biopsy, and observed a treatment failure rate of $30 \%$ in the cryotherapy group versus $19 \%$ in the biopsy group after 24 months. On the other hand, a randomized study found no difference after 12 months, between 86 women who had had a cone biopsy for HSIL and 80 women treated by cryotherapy [14]. In the meta-analysis of Debeaudrap et al. [8] a sub-group analysis limited to HIV-positive women treated for HSIL showed a higher risk of treatment failure after cryotherapy (21.6\%) compared to cone biopsy (12.6\%).

Although the size of the removed cones was similar between HIV-positive and -negative women in our study, the margins were more often positive in HIV-positive women (37 versus 16\%). These observations were also found in other studies and support the idea of the presence of more extensive lesions in HIV-positive women compared to HIV-negative ones and could explain treatment failure [8].

Comparing HIV women with treatment failure and those without, we observed that shorter duration of cumulative undetectable HIV VL, or VL > 50 copies/ $\mathrm{ml}$ at the time of HSIL treatment, higher maximum VL between time of HIV diagnosis and cervical excision, a lower $\mathrm{CD} 4 / \mathrm{CD} 8$ ratio, lower nadir CD4 count and lower CD4 count at time of dysplasia were associated with higher rates of treatment failure. Atemnkeng et al. [15] evaluated the association between cART, viral load, CD4 count and detection of CIN $2+$ at follow-up. They 
observed that cART intake was associated with a reduced risk of CIN 2 + detection at follow-up, after excisional procedures, but not after ablative treatment, as well as in cART users with undetectable VL. They did not report an association between CD4 count at the time of dysplasia discovery and treatment failure. Two studies, within the meta-analysis of Atemnkeng, reported that a nadir CD4 count of more than 350 cells $/ \mathrm{mm}^{3}$ was associated with a $65 \%$ decreased risk of CIN $2+$ detection at follow-up.

At this time, the recommendations for HSIL management between HIV positive and negative women do not differ. The World Health Organization and ASCCP recommend excision over ablation in settings where both are available, but ablation is acceptable for young women in satisfactory clinical conditions (when pregnancy is desired, the colposcopy image is satisfactory and followup compliance is probable) $[10,16]$.

In our clinical practice, managing young, HIV positive women with a recent diagnosis of HSIL and control of their HIV infection presents a challenge, when weighing the potential, future obstetric risk against the risk of cervical lesion progression.

Indeed, despite the scaling up access of cART and easier regimens (one pill regimen with less side effects), invasive cervical cancer is still more prevalent in HIVpositive women [17]. A recent study reported data regarding cervical cancer risk, in women living with HIV across four continents. Being older than 50 and having a low CD4 count at the time of cART initiation, were risk factors for invasive cervical cancer development [17]. Our data are in agreement, since we found that cervical treatment failure was 3.5 times higher in women who had a viral load exceeding 50 copies $/ \mathrm{ml}$ at the time of the HSIL diagnosis.

We therefore suggest that cervical cancer management guidelines must be adapted for HIV-positive women. Excisional treatment should be preferred over ablative therapy, particularly in women with uncontrolled viral load at the time of the HSIL diagnosis. Ablative therapy could be an option for young women who have an undetectable viral load and CD4 + lymphocytes higher than 500 cells $/ \mathrm{mm}^{3}$ and have a desire of pregnancy.

In our study, few patients were vaccinated against HPV, even though it is known that HPV vaccination at the time of the cone biopsy, reduces the risk of recurrence of HSIL in HIV-negative women $[18,19]$. Although this has not yet been established in HIV-positive women, similar results, for them, might be found. In view of the high recurrence rate, studies should be performed to find an answer to this question. Pending their results, vaccination should be proposed for both HIV-negative and positive women.
Our study has several limitations, mostly related to the retrospective design and the real clinical setting of a cohort, so we were not able to match the two groups for all of the possible confounding factors. Most of the women in the HIV group were Black African patients and most of our HIV negative patients were of European origin. This is an important confounder that we could not correct for a number of variables, results were occasionally unavailable. Not all women had the same follow-up schedule. In some cases, distinguishing persistence from a re-infection may have been erroneous. Moreover, the guidelines on ART and the identification of high risk HPV (HR HPV) has evolved over time. Finally, vaccination has only been available since 2008 in Belgium. However, the large cohort with a long follow up, the detailed HIV infection surrogates markers and the real-life follow up setting contribute to the strengths of this study.

\section{Conclusion}

HIV-positive women have a higher rate of treatment failure for HSIL than do HIV-negative women. Ablative therapy is associated with a higher risk of treatment failure, especially in women with poor control of their HIV infection and should therefore be avoided for these patients. The management and the follow- up of HSIL's guidelines in this high-risk population should be adapted consequently: for HIV-positive women with uncontrolled viral load, excisional treatment should be the preferred therapy, whereas for women with undetectable viral load, CD4 + lymphocytes higher than 500 cells $/ \mathrm{mm}^{3}$ and with a desire of pregnancy, ablative therapy may be considered.

\section{Authors' contributions}

Christine Gilles: conceptualization, methodology, validation, formal analysis, investigation, writing-original draft, visualization, supervision. Maude Velghelenelle: formal analysis, investigation, writing-original draft. Yannick Manigart: conceptualization, visualization, supervision. Deborah Konopnicki: conceptualization, writing-review and editing, supervision, project administration. Serge Rozenberg: conceptualization, validation, formal analysis, writing-review and editing, supervision, project administration. All authors read and approved the final manuscript

Funding

No Funding support this research.

Availability of data and materials Not applicable.

Code availability

Not applicable. 


\section{Declarations}

Ethics approval and consent to participate

The study was approved on March 20th, 2018 by the Saint-Pierre University Hospital Ethical Review Board (nr CE/18-03-08). Consent to participate is not applicable.

\section{Consent for publication}

Not applicable.

\section{Competing interests}

The authors declare that they have no known competing financial interests or personal relationships that could have appeared to influence the work reported in this paper.

\section{Author details}

1 Department of Obstetrics and Gynecology, Saint Pierre University Hospital Brussels, Université Libre de Bruxelles (ULB), Free University of Brussels (ULBVUB), 322 Rue Haute, 1000 Brussels, Belgium. ${ }^{2}$ Infectious Disease Department, Saint-Pierre University Hospital Brussels, Université Libre de Bruxelles (ULB), Brussels, Belgium.

Received: 11 November 2020 Accepted: 13 July 2021

Published online: 23 July 2021

\section{References}

1. UNAIDS. Aids by the Numbers 2018. https://www.unaids.org/en/resou rces/documents/2019/aids-by-the-numbers. Accessed 2 June 2020

2. https://www.who.int/cancer/prevention/diagnosis-screening/cervicalcancer/en/. Accessed 2 June 2020.

3. From the Centers for Disease Control and Prevention. 1993 revised classification system for HIV infection and expanded surveillance case definition for AIDS among adolescents and adults. JAMA. 1993;269(6):729.

4. Du P. Human papillomavirus infection and cervical cancer in HIV+ women. Cancer Treat Res. 2019;177:105-29.

5. Liu G, Sharma M, Tan N, Barnabas RV. HIV-positive women have higher risk of human papilloma virus infection, precancerous lesions, and cervical cancer. AIDS. 2018;32(6):795-880.

6. Denslow SA, Rositch AF, Firnhaber C, Ting J, Smith JS. Incidence and progression of cervical lesions in women with HIV: a systematic global review. Int J STD AIDS. 2014;25(3):163-77.

7. Gilles C, Manigart Y, Konopnicki D, Barlow P, Rozenberg S. Management and outcome of cervical intraepithelial neoplasia lesions: a study of matched cases according to HIV status. Gynecol Oncol. 2005;96:112-8.

8. Debeaudrap P, Sobngwi J, Tebeu PM, Clifford GM. Residual or recurrent precancerous lesions after treatment of cervical lesions in human immunodeficiency virus-infected women: a systematic review and metaanalysis of treatment failure. Clin Infect Dis. 2019;69(9):1555-65.
9. Altice F, Evuarherhe O, Shina S, Carter G, Beaubrun AC. Adherence to HIV treatment regimens: systematic literature review and meta-analysis. Patient Prefer Adherence. 2019;13:475-90. https://doi.org/10.2147/PPA. S192735 (PMID: 31040651).

10. Santesso N, Mustafa RA, Schünemann HJ, et al. World Health Organization Guidelines for treatment of cervical intraepithelial neoplasia 2-3 and screen-and-treat strategies to prevent cervical cancer. Int J Gynaecol Obstet. 2016;132(3):252-8. https://doi.org/10.1016/j.ijgo.2015.07.038.

11. D'Alessandro P, Arduino B, Borgo M, et al. Loop electrosurgical excision procedure versus cryotherapy in the treatment of cervical intraepithelial neoplasia: a systematic review and meta-analysis of randomized controlled trials. Gynecol Minim Invasive Ther. 2018;7:145-51.

12. Chirenje ZM, Rusakaniko S, Akino V, Munjoma M, Mlingo M. Effect of HIV disease in treatment outcome of cervical squamous intraepithelial lesions among Zimbabwean women. J Low Genit Tract Dis. 2003;7:16-21.

13. Greene SA, De Vuyst H, John-Stewart GC, et al. Effect of cryotherapy vs loop electrosurgical excision procedure on cervical disease recurrence among women with HIV and high-grade cervical lesions in Kenya: a randomized clinical trial. JAMA. 2019;322:1570.

14. Smith JS, Sanusi B, Swarts A, et al. A randomized clinical trial comparing cervical dysplasia treatment with cryotherapy versus loop electrosurgical excision procedure in HIV-seropositive women from Johannesburg, South Africa. Am J Obstet Gynecol. 2017;217:e1-11.

15. Atemnkeng N, Desmond A, de Sanjose S, Mayaud P. Kelly H Antiretroviral therapy and detection of high-grade cervical intraepithelial neoplasia (CIN2+) at post-CIN management follow-up among women living with HIV: a systematic review and meta-analysis. Clin Infect Dis. 2020. https:// doi.org/10.1093/cid/ciaa238.

16. Perkins RB, Guido RS, Castle PE, et al. 2019 ASCCP Risk-Based Management Consensus Guidelines Committee. J Low Genit Tract Dis. 2020;24(2):102.

17. Rohner E, Bütikofer L, Schmidlin K, et al. Cervical cancer risk in women living with HIV across four continents: a multicohort study. Int J Cancer. 2020;146:601-9.

18. Ghelardi A, Parazzini F, Martella F, Pieralli A, Bay P, Tonetti A, Svelato A, Bertacca G, Lombardi S, Joura EA. SPERANZA project: HPV vaccination after treatment for CIN2. Gynecol Oncol. 2018;151:229-34. https://doi. org/10.1016/j.ygyno.2018.08.033.

19. Kang WD, Choi HS. Kim SM Is vaccination with quadrivalent HPV vaccine after loop electrosurgical excision procedure effective in preventing recurrence in patients with high-grade cervical intraepithelial neoplasia (CIN2-3)? Gynecol Oncol. 2013;130(2):264-8. https://doi.org/10.1016/j. ygyno.2013.04.050 (PMID: 23623831).

\section{Publisher's Note}

Springer Nature remains neutral with regard to jurisdictional claims in published maps and institutional affiliations.
Ready to submit your research? Choose BMC and benefit from:

- fast, convenient online submission

- thorough peer review by experienced researchers in your field

- rapid publication on acceptance

- support for research data, including large and complex data types

- gold Open Access which fosters wider collaboration and increased citations

- maximum visibility for your research: over $100 \mathrm{M}$ website views per year

At BMC, research is always in progress.

Learn more biomedcentral.com/submissions 